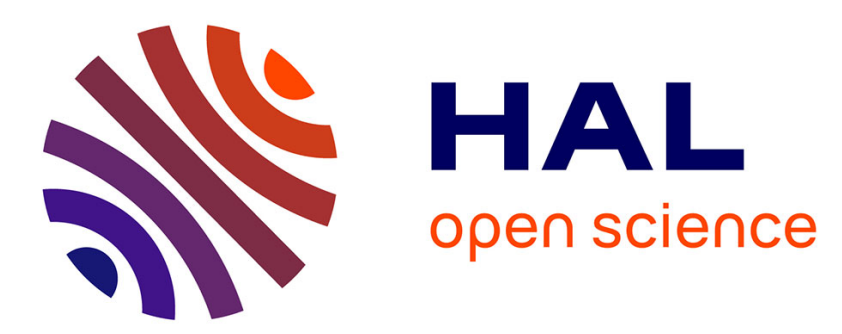

\title{
Apports de l'analyse temporelle des otoémissions acoustiques provoquées transitoires à l'identification de leurs sources
}

P. Avan, H. Wit

\section{- To cite this version:}

P. Avan, H. Wit. Apports de l'analyse temporelle des otoémissions acoustiques provoquées transitoires à l'identification de leurs sources. Journal de Physique IV Proceedings, 1994, 04 (C5), pp.C5-403-C5406. 10.1051/jp4:1994584 . jpa-00253079

\section{HAL Id: jpa-00253079 https://hal.science/jpa-00253079}

Submitted on 1 Jan 1994

HAL is a multi-disciplinary open access archive for the deposit and dissemination of scientific research documents, whether they are published or not. The documents may come from teaching and research institutions in France or abroad, or from public or private research centers.
L'archive ouverte pluridisciplinaire HAL, est destinée au dépôt et à la diffusion de documents scientifiques de niveau recherche, publiés ou non, émanant des établissements d'enseignement et de recherche français ou étrangers, des laboratoires publics ou privés. 


\title{
Apports de l'analyse temporelle des otoémissions acoustiques provoquées transitoires à l'identification de leurs sources
}

\author{
P. AVAN et H.P. WIT*
}

Laboratoire de Biophysique, Faculté de Médecine, Université d'Auvergne, BP. 38, 63001 Clermont-Ferrand cedex, France

* Institute of Audiology, University Hospital, P.O. Box 30.001, 9700 RB Groningen, The Netherlands

Summary: Transient-evoked otoacoustic emissions (t-EOE) are of growing importance in clinical routine as an objective tool for screening hearing losses due to cochlear dysfunction. For this purpose, their presence or absence is thought to be a reliable criterion. Apart from such applications, the question of their generation and propagation in a normal or impaired cochlea remains challenging. In particular, it seems that several of their properties are sensitive to the status of the whole cochlea, thus if it was confirmed, it would not be straightforward to predict the alterations of t-EOE components as a function of audiometric data, and the reverse would be even more delicate. The goal of this work was to substantiate the hypothesis that tEOE properties depend the whole cochlear status by analysing their temporal patterns in human ears, with either irreversible audiometric alterations attributed to noise-induced hearing loss, or reversible changes in basal cochlear function produced by high-frequency masking tones. The temporal patterns examined by wavelet analysis were conspicuously dependent on the cochlear status at high frequencies. In the first experiment (NIHL), EOE patterns enabled to predict whether or not the audiogram was normal around 8 $\mathrm{kHz}$. With high-frequency masking, very complex changes in temporal patterns were observed. These results suggest that EOE and hearing loss are correlated in a complex manner.

\section{INTRODUCTION.}

Pour une utilisation clinique en vue du dépistage des surdités d'origine cochléaire, les otoémissions acoustiques provoquées (OEAP), maintenant largement utilisées $[1,2]$, peuvent être analysées de manière binaire: présentes ou absentes (revue très complète dans [3]). En revanche, une meilleure compréhension de leurs mécanismes de genèse et de propagation, non encore clairement élucidés, nécessite des analyses plus fines, notamment pour déterminer comment les composantes fréquentielles des OEAP se modifient soit lors d'une perte auditive, soit lors d'une modification réversible de la fonction cochléaire, telle que celles induites par un son masquant. Les amplitudes globales des OEAP ont déjà été étudiées dans de telles circonstances $[4,5,6,7]$. Dans les deux cas, elles se sont révélées sensibles à l'état global de la cochlée basale. Ceci peut paraître paradoxal dans la mesure où on considère en général les OEAP comme résultant de mécanismes associés au fonctionnement des cellules ciliées externes de l'organe de Corti, elles même supposées intervenir très localement dans la micromécanique cochléaire.

Le but de ce travail est de tenter de confirmer et documenter ces modifications en examinant l'allure temporelle des composantes d'EOAE humaines dans des circonstances analogues, avec l'hypothèse de travail que les EOAE résultent d'activités réparties le long de la cochlée et interférant de manière complexe [8] et désordonnée [9]. On pourrait s'attendre à ce qu'une pathologie ou une mise hors circuit par un masquage de haute fréquence simplifie ces interférences en diminuant le nombre de sites actifs émetteurs d'OEAP, ou, alternativement, à ce que la propagation rétrograde des multiples composantes d'OEAP soit perturbée en traversant des sites pathologiques ou masqués à la base de la cochlée. 
Les conséquences cliniques d'une telle étude pourraient être intéressantes dans la mesure où la mise en évidence de modifications systématiques des OEAP lorsque la partie la plus basale de la cochlée est anormale pourrait permettre d'étendre la gamme fréquentielle utilisable et, peut-être, la sensibilité de ce test à des atteintes précoces.

\section{MATÉRIEL ET MÉTHODES}

\subsection{Expérience $n^{\circ} 1$}

La première partie du travail a été effectuée sur 45 oreilles de sujets adultes venant consulter pour une surdité après exposition professionnelle au bruit. Après otoscopie et impédancemétrie pour éliminer une atteinte transmissionnelle, leur audiogramme tonal était complété par une audiométrie automatique de Békésy par balayage entre 0,25 et $8 \mathrm{kHz}$. Tous ces sujets avaient des potentiels évoqués du tronc cérébral en faveur d'une atteinte strictement endocochléaire, présumée due à des lésions électives des cellules ciliées externes de l'organe de Corti. Les sujets se répartissaient en deux catégories selon la forme de leur audiogramme quand la fréquence augmente (en pente régulière, 29 oreilles désignées par HF-, ou bien en encoche avec audition normale à $6-8 \mathrm{kHz}, 17$ oreilles désignées par $\mathrm{HF}+$ ).

Leurs OEAP étaient détectées par un appareil ILO88 (Otodynamics) en mode standard non-linéaire (clics de $85 \mathrm{~dB}$ p.eq. SPL; voir par exemple [10]). Elles étaient ensuite analysées par décomposition en ondelettes (voir [9]) permettant d'obtenir un diagramme temps/fréquence pour l'enveloppe de chaque composante fréquentielle (pas temporel: $0.3 \mathrm{~ms}$, pas fréquentiel: $100 \mathrm{~Hz}$ ). La forme des enveloppes des réponses autour de 2 fréquences particulières était étudiée: à $1 \mathrm{kHz}$ et à la plus haute fréquence détectable des OEAP, soit $f_{\max }$. Le plus souvent, cette fréquence était inférieure d'une demie à une octave au bord "basse fréquence" de l'encoche audiométrique, définie avec une précision de quelques centaines de $\mathrm{Hz}$ à l'audiométrie par balayage.

\subsection{Expérience $\mathrm{n}^{\circ} 2$}

5 sujets jeunes ont été inclus dans cette série de mesures après avoir subi les mêmes contrôles (cette fois tous normaux) que les sujets de l'expérience 1. L'enregistrement des OEA provoquées par des clics (45 à $55 \mathrm{~dB}$ p.eq.SPL) était réalisé à partir d'un système constitué d'une sonde de réalisation personnelle (microphone Sennheiser, haut parleur miniature Knowles). Le signal d'OEAP était préamplifié ( $20 \mathrm{~dB})$ puis traversait deux filtres en série (Krohn-Hite $48 \mathrm{~dB}$ /octave passe bande $0.7-2 \mathrm{kHz}$ ) et deux amplificateurs (Philips, $20 \mathrm{~dB}$ ) avant d'être sommé (1024 fois) dans un moyenneur digital Datalab 16 bit. Un mélangeur permettait d'ajouter un masque au clic générateur des OEAP, soit un son pur à $0.75 \mathrm{ou} 2 \mathrm{kHz}$, soit un bruit filtré passe haut ( $>2 \mathrm{kHz}$; filtre Krohn-Hite $48 \mathrm{~dB} /$ octave) de densité spectrale d'énergie constante entre 2 et $13 \mathrm{kHz}$.

Le niveau acoustique des sons masquants devait rester inférieur ou égal à $50 \mathrm{~dB}$ SPL de manière à ne pas entraîner de surcharge ou de saturation au niveau du système détecteur. Le niveau acoustique des clics devait être fixé aussi bas que possible compte tenu du seuil de détection de chaque sujet pour permettre au son masquant d'être aussi intense, donc efficàce, que possible par rapport au stimulus. Les OEAP ainsi obtenues avant et pendant l'application des sons masquants (essentiellement de basse fréquence compte tenu du filtrage choisi dans la chaîne de recueil) étaient ensuite comparées par analyse en ondelette (comme pour l'expérience $n^{\circ} 1$ ). Les phases respectives des différentes composantes fréquentielles étaient également comparées.

\section{RÉSULTATS.}

\subsection{Expérience $n^{\circ} 1$}

\subsubsection{Cas des oreilles de type $H F+$}

L'otoémission de fréquence $f_{\max }$ avait une enveloppe complexe (présentant plusieurs maximums entre 2.5 et $20 \mathrm{~ms}$ ) dans 16 cas sur 17 . Celle de fréquence proche de $1 \mathrm{kHz}(\mathrm{a} 0,15 \mathrm{kHz}$ près) présentait toujours une enveloppe complexe. 


\subsubsection{Cas des oreilles de type $H F$ -}

Le comportement des OEAP était totalement différent de celui des observé dans les oreilles $\mathrm{HF}+$ à $\mathrm{f}_{\max } \cdot$ En effet, l'enveloppe de cette composante était simple (un seul maximum entre 2.5 et $20 \mathrm{~ms}$ ) dans 26 cas sur 29. Toutefois, un comportement identique à celui des oreilles $\mathrm{HF}+$ était noté à $1 \mathrm{kHz}$ (sauf toutefois pour les 7 cas où $f_{\max }$ ne différait de $1 \mathrm{kHz}$ que de moins de $0.5 \mathrm{kHz}$ ), c'est-à-dire la présence d'une structure complexe des enveloppes.

\subsubsection{Cas d'oreilles normales}

Afin de pouvoir disposer d'un point de comparaison et interpréter ces résultats sur le base de ce qui se passe dans des oreilles normales, 40 oreilles normales ont été étudiés à titre de contrôle, aux fréquences 1 et 2 $\mathrm{kHz}$. Cette dernière valeur a été choisie car plus de $50 \%$ des sujets atteints de perte auditive avaient une valeur de $f_{\max }$ proche de $2 \mathrm{kHz}$. Pour les deux fréquences, les enveloppes étaient de forme complexe dans $42,5 \%$ et $47.5 \%$ des cas (respectivement). Ainsi, globalement, les répartitions des oreilles entre les catégories "enveloppe simple" et "enveloppe complexe" présentaient une liaison très significative avec le type d'audiogramme (test du $\chi^{2} ; p<0,0001$ )

\subsection{Expérience $\mathrm{n}^{\circ} 2$}

\subsubsection{Cas d'un masqueur haute fréquence}

La comparaison des enveloppes aussi bien que des phases en l'absence et en présence de masqueur (bruit filtré passe-haut ou son pur à $2 \mathrm{kHz}$ ) a montré des changements considérables de la forme des composantes d'OEAP, associés à la diminution d'amplitude déjà observée [7]. Notamment, l'enveloppe de ces composantes présentait une structure complètement différente en présence de masqueur, avec augmentation ou diminution du nombre de maximums et changements des latences de plusieurs ms. Au contraire de l'expérience précédente, aucune règle systématique n'a pu être mise en évidence (il est vrai sur 5 sujets). De plus, les changements observés n'évoluaient pas de manière monotone lors de l'augmentation progressive du niveau du masqueur.

\subsubsection{Cas d'un masqueur basse fréquence}

Globalement, les mêmes caractéristiques que celles décrites dans la section précédente ont été observées, avec toutefois une diminution plus rapide de l'amplitude des échos lorsque le niveau acoustique du masqueur augmentait.

\section{DISCUSSION.}

Les résultats obtenus dans les deux cas apportent deux indications complémentaires. D'une part, une modification irréversible des seuils auditifs haute fréquence (autour de $4 \mathrm{kHz}$ ), vraisemblablement due à des lésions électives des cellules ciliées externes de l'organe de Corti, se traduit par la présence de patterns temporels très particuliers pour les composantes d'otoémissions de basse fréquence. Plusieurs travaux ont démontré que, loin d'être sensibles uniquement à l'état cochléaire local correspondant à leur fréquence, les otoémissions provoquées transitoires ont une amplitude fonction de l'ensemble de l'état cochléaire basal (revue dans [5]). On voit ici que cette diminution d'énergie des composantes d'OEAP de basse fréquence n'est pas uniformément répartie en fonction du temps. Ceci peut correspondre à ce que Zwicker [8] mettait en évidence dans son modèle électronique en arrêtant le fonctionnement de certaines sections actives. Cependant, nos résultats ne peuvent être expliqués par un tel modèle qu'à la condition d'étendre la contribution cochléaire aux composantes d'OEAP jusqu'à l'extrême base, ce qui semble difficilement conciliable avec les modèles d'accordage cochléaire, suggérant que les $\mathrm{CCE}$ jouent un rôle amplificateur très local.

Dans le cadre d'une telle hypothèse, on pourrait proposer que les composantes à $f_{\text {max }}$ des oreilles HF-, ne résultant plus que de sources très réduites et localisées, ont ainsi une structure temporelle très simplifiée par rapport à ce qui ets observé chez des sujets normaux, tandis que celles des oreilles de type HF+ proviendraient de battements entre sources d'extension limitées, l'une au voisinage du point codant pour $\mathrm{f}_{\text {max }}$, l'autre à l'extrême base. Une hypothèse alternative est que les oreilles $\mathrm{HF}+$, la présence d'une nette discontinuité d'impédance ("passive") au milieu de la cochlée entraîne l' "apparition" de battements nets. 
Bien sûr, le terme "apparition" est purement spéculatif en l'absence de tracés témoins dans la même oreille avant lésion. C'est pour cette raison que nous nous sommes intéressés au masquage (cette fois réversible) par des sons de haute fréquence, dans le but de simuler la situation observée dans une surdité cochléaire. La même idée avait été utilisée par Sutton [6] ainsi que Brass et Kemp [7] qui n'ont décrit que des effets de réduction d'amplitude des OEAP de basse fréquence par des masqueurs haute fréquence. Nos résultats actuels montrent un effet très complexe des sons masquants sur le pattern temporel des OEAP. Le masquage n'avait sans doute pas un niveau assez élevé pour simuler une surdité. L'existence de modifications aussi nettes malgré la différence entre fréquences masquantes et fréquences d'OEAP reste à expliquer dans le cadre des modèles de micromécanique cochléaire, de même pour la notion de "latence" d'OEAP, déjà discutée dans un récent travail [10].

REMERCIEMENTS. Ce travail a été financé grâce à une subvention attribuée aux deux auteurs dans le cadre des accords INSERM / NWO en 1993-94. Pour la première partie de l'étude, Paul Avan a également bénéficié d'une subvention de recherche de l'Institut Electricité-Santé en 1992-93.

\section{BIBLIOGRAPHIE.}

[1] Kemp D.T., JAcoust.Soc.Am. 64, (1978), 1386-1391.

[2] Kemp D.T., Ryan S., Seminars in Hearing 14-1 (1993), 30-45.

[3] Probst R., Lonsbury-Martin B.L., Martin G.K., J.Acoust.Soc.Am. 89, (1991), 2027-2067.

[4] Avan P., Bonfils P., Loth D., Narcy Ph., Trotoux J., Hearing Res. 52, (1991), 99-112.

[5] Avan P., Bonfils P., Loth D., Teyssou M., Menguy C., Prog.Brain Res. 97, (1993), 67-75.

[6] Sutton G.J., Acustica 58, (1985), 57-63.

[7] Brass D., Kemp D.T., J.Acoust.Soc.Am. 93-2 (1993), 920-939.

[8] Zwicker E., "Cochlear mechanisms, structures, function and models", J.P.Wilson and D.T.Kemp (eds), Plenum (1989), pp.359-366.

[9] Wit H.P., van Dijk P., Avan P., Hearing Res. (1994) (to be published)

[10] Avan P., Bonfils P., Loth D., Wit H.P., Hearing Res. 70, (1993), 109-120.
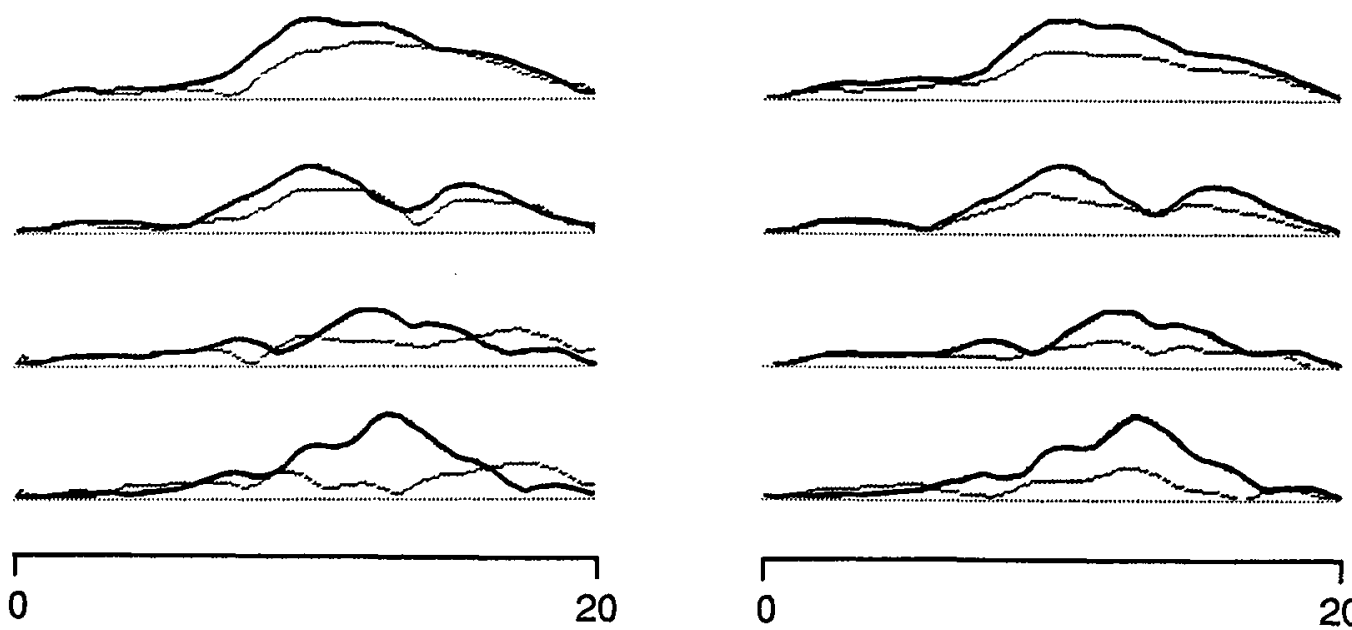

20 (ms)

figure 1: décomposition en ondelettes des OEAP d'une oreille en dessous de $2 \mathrm{kHz}$ (entre 1 et $1,6 \mathrm{kHz}$ par pas de $200 \mathrm{~Hz}$ ). En trais épais, absence de masquage; le niveau du clic est de $0 \mathrm{~dB}$ SL. En traits fins, OEAP en présence d'un son masquant de $2 \mathrm{kHz}$ à $45 \mathrm{~dB}$ SPL (gauche) puis $50 \mathrm{~dB}$ SPL (droite). On constate une diminution d'amplitude, des changements dans la "latence" et le nombre de maximums. 